\title{
b-Colouring of Central Graphs
}

\author{
Dr.K.Thilagavathi \\ Associate Professor, \\ Department of Mathematics, \\ Kongunadu Arts \& Science College, \\ Coimbatore-641 029, India.
}

\author{
Mrs. D.Vijayalakshmi \\ Assistant Professor and Head, \\ Department of Mathematics CA, \\ Kongunadu Arts \& Science College, \\ Coimbatore-641 029, India.
}

\author{
Mr. N.Roopesh \\ Research Scholar, \\ Department of Mathematics, \\ Kongunadu Arts \& Science College, \\ Coimbatore-641 029, India.
}

\begin{abstract}
In this paper we discuss about the b-colouring and b-chromatic number of $\mathrm{C}\left(\mathrm{C}_{\mathrm{n}}\right), \mathrm{C}\left(\mathrm{K}_{\mathrm{m}, \mathrm{n}}\right)$ and $\mathrm{C}(\mathrm{P} n)$.
\end{abstract}

\section{Keywords}

Central graph, b-colouring and b-chromatic number.

\section{INTRODUCTION}

Let $\mathrm{G}$ be a finite undirected graph with no loops and multiple edges. The central graph $\mathrm{C}(\mathrm{G})$ [10] of a graph $\mathrm{G}$ is obtained by subdividing each edge of $G$ exactly once and joining all the non-adjacent vertices of $G$. By definition $P_{c}(G)=p+q$. For any $(p, q)$, graph there exists exactly $p$ vertices of degree $(p-1)$ and $q$ vertices of degree 2 in $C(G)$.

The b-chromatic number [6] of a graph was introduced by R.W.Irving and D.F.Manlove when considering minimal proper colouring with respect to a Partial order defined on the set of all partition of vertices of graph. The b-chromatic number of a graph $\mathrm{G}$, denoted by $\varphi(\mathrm{G})$, is the largest positive integer $\mathrm{t}$ such that there exists a proper coloring for $G$ with $t$ colors in which every color class contains at least one vertex adjacent to some vertex in all the other colour classes such a colouring is called a b-colouring.

\section{THE b-COLOURING OF C $\left(K_{m, n}\right)$}

\subsection{Theorem}

For any complete bipartite graph $\mathrm{C}\left(\mathrm{K}_{\mathrm{m}, \mathrm{n}}\right), \varphi\left(\mathrm{C}\left(\mathrm{K}_{\mathrm{m}, \mathrm{n}}\right)\right)=$ $\mathrm{n}+\left[\frac{m}{2}\right]$ where $\mathrm{m} \leq 6$.

\section{Proof}

Consider the complete bipartite graph $\mathrm{K}_{\mathrm{m}, \mathrm{n}}$ with bipartation $(\mathrm{X}, \mathrm{Y})$ where $X=\left\{v_{1}, v_{2}, \ldots ., v_{n}\right\}$ and $Y=\left\{u_{1}, u_{2}, \ldots ., u_{n}\right\}$ in $C\left(K_{m, n}\right)$. Let $\mathrm{v}_{\mathrm{i}, \mathrm{j}}$ represents the newly introduced vertex in the edge joining $\mathrm{v}_{\mathrm{i}}$ and $\mathrm{u}_{\mathrm{j}}$. Now assign a colouring to the vertices of $\mathrm{C}\left(\mathrm{K}_{\mathrm{m}, \mathrm{n}}\right)$ as follows. Assign the colour $c_{i}$ to $v_{i}$ for $i=1,2, \ldots$, n. since $\left\langle v_{i}, i=1,2, \ldots, n\right\rangle$ is a complete graph, this colouring will be a bcolouring. Give the colour $\mathrm{c}_{\mathrm{n}+\mathrm{i}}$ to $\mathrm{u}_{\mathrm{i}}$ for $\mathrm{i}=1,2, \ldots,\left[\frac{m}{2}\right]$, now the vertex which has been coloured as $c_{n+i}$ cannot realises the colour $c_{n+i}$ to $u_{i}$. In order to overcome this, we should colour the $v_{i, j}, s$, $\mathrm{i} \neq \mathrm{n}$ as $\mathrm{c}_{\mathrm{i}+1}$ and $\mathrm{v}_{\mathrm{i}, \mathrm{j}}{ }^{\mathrm{s}} \mathrm{s}, \mathrm{i}=\mathrm{n}$ as $\mathrm{c}_{1}$ where $\mathrm{j} \leq\left[\frac{m}{2}\right]$. Again the introduction of new colours, namely $c_{n+i}$ made the colouring of $v_{i}$, $\mathrm{i}=1,2, \ldots ., \mathrm{n}$ is no more $\mathrm{b}$-chromatic. To make this colouring $\mathrm{a} b$ chromatic one, we should colour $\mathrm{v}_{\mathrm{i}, \mathrm{j}}, \mathrm{j}=\left[\frac{m}{2}\right]+\mathrm{k}, \mathrm{k}=1,2$, $\ldots . .\left[\frac{m}{2}\right]$ as $\mathrm{c}_{\mathrm{n}+\mathrm{k}}$. Thus to colour the remaining vertices in $\mathrm{u}_{\mathrm{i}, \mathrm{i}} \mathrm{i}>$ $\left[\frac{m}{2}\right]$, for this vertices we cannot assign any new colours because all the $v_{i j}$ 's which are adjacent to any $u_{i}$ is of same colour and those $u_{i}$ 's are not at all adjacent with any of the $c_{i}$ coloured vertices. Hence, by colouring procedure the above said colouring is a b-chromatic colouring and furthermore it is the maximum colouring possible. Hence $\varphi\left(\mathrm{C}\left(\mathrm{K}_{\mathrm{m}, \mathrm{n}}\right)\right)=\mathrm{n}+\left[\frac{m}{2}\right]$.

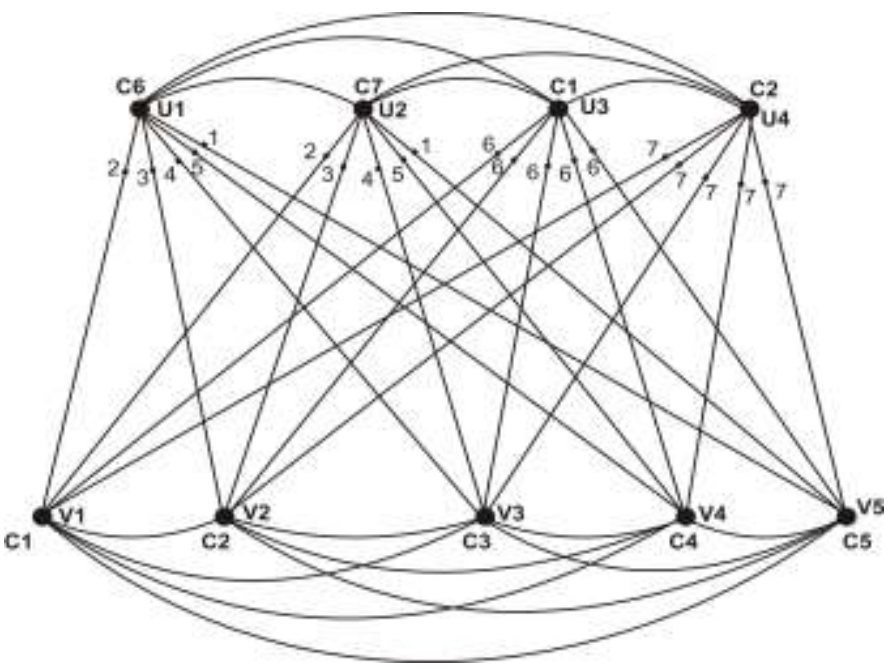

Figure $1: \varphi\left[C\left(K_{4,5}\right)\right]=7$

\section{THE b-COLOURING OF [C(Cn)]}

\subsection{Theorem}

For any cycle $C_{n}$ of length $n \geq 5, n=5 x+r$, 


$$
\varphi\left[\begin{array}{ll}
C & C_{n}
\end{array}\right]= \begin{cases}n-x+1 & \text { when } \mathrm{r} \neq 0 \\
n-x & \text { when } \mathrm{r}=0\end{cases}
$$

\section{Proof}

Let $C_{n}$ be any cycle of length $n$ with vertices $v_{1}, v_{2} \ldots v_{n}$. Let $v_{i j}$ represents the newly introduced vertex in the edge connecting $\mathrm{v}_{\mathrm{i}}$ and $v_{j}$. Now in $C\left(C_{n}\right)$ we can note that the vertex $v_{i}$ is adjacent with all the vertices except the vertices except $v_{i+1}$ and $v_{i-1}$ for $i=$ $2,3,4,5, \ldots \mathrm{n}-1 . \mathrm{v}_{1}$ is adjacent with all the vertices except $\mathrm{v}_{2}$ and $\mathrm{v}_{\mathrm{n}}$ and $\mathrm{v}_{\mathrm{n}}$ is adjacent with all the vertices except $\mathrm{v}_{\mathrm{n}-1}$ and $\mathrm{v}_{1}$. Consider a blind colouring of $\mathrm{C}\left(\mathrm{C}_{\mathrm{n}}\right)$ as follows. Assign the colour $\mathrm{c}_{\mathrm{i}}$ to $\mathrm{v}_{\mathrm{i}}$ for $\mathrm{i}=1,2, \ldots ., \mathrm{n}$. Due to the above said non-adjacency of $v_{i}^{\prime}$ s this colouring will not produce a b-colouring. Thus to make it a b-colouring, we should assign a proper colour to $\mathrm{v}_{\mathrm{ij}}$ 's. Consider an arbitary vertex $v_{i}$, but $v_{i}$ is not adjacent with $v_{i+1}$ and $\mathrm{v}_{\mathrm{i}-1}$. To realize the colour $\mathrm{c}_{\mathrm{i}}$ we should colour $\mathrm{v}_{\mathrm{i}, \mathrm{i}+1}$ as $\mathrm{c}_{\mathrm{i}-1}$ and $\mathrm{v}_{\mathrm{i}, \mathrm{i}-1}$ as $c_{i+1}$. Thus $v_{i}$ will realise the colour $c_{i}$. Now take the vertex $v_{i+1}$, which is coloured as $c_{i+1}$. In order to realise the colour $c_{i+1}$, we should colour two neighbours of $v_{i+1}$ as $c_{i+1}$ and $c_{i}$ but the previous colouring of $\mathrm{v}_{\mathrm{i}}$ had left out only one vertex namely $v_{i+1, i+2}$ to be coloured. Thus realisation of $c_{i+1}$ is not possible. Similar situation will occur if we are proceeding with $\mathrm{v}_{\mathrm{i}-1}$ too. This shows that assigning different colours to $\mathrm{v}_{\mathrm{i}}$ 's is not possible. i.e. there should be repetation of colours. A close examination will reveal that there should be minimum of $\left\lceil\frac{n}{5}\right\rceil$ repetations. Thus we will assign a colouring to $C\left(C_{n}\right)$ as follows.

Case: 1

When $\mathrm{r}=0$, assign the colour $\quad c_{i-\left[\frac{i}{5}\right]}$ to the vertex $\mathrm{v}_{\mathrm{i}}$ for $\mathrm{i}=1,2$, ...., n. Here only the repeated colour vertex realises its own colour but for the remaining vertex it is not possible. So the above colouring does not produce a b-colouring. To make it a bcolouring, we assign a proper colouring $\mathrm{v}_{\mathrm{i}, \mathrm{j}}$ 's as follows. For $\mathrm{i}=1$, $2, \ldots, \mathrm{n}-1$ and $\mathrm{i} \equiv 2,3,4(\bmod 5)$ assign the colour $c_{i-\left(\left[\frac{i}{5}\right]_{-1}\right]}$ to the vertex $\mathrm{v}_{\mathrm{i}, \mathrm{i}+1}$ otherwise assign the colour $c_{i-\left(\left[\frac{i}{5}\right]+2\right)}$ to $\mathrm{v}_{\mathrm{i}, \mathrm{i}+1}$. Now all $v_{i}$ 's for $i=1,2, \ldots ., n$ realises its own colour $c_{i}$. Hence by the colouring procedure it is the maximum colouring.

\section{Case: 2}

When $\mathrm{r} \neq 0$, for $\mathrm{i}=1,2, \ldots, \mathrm{n}-1$ assign the colour $c_{i-\left[\frac{i}{5}\right]}$ to the vertex $v_{\mathrm{i}}$. Here also only the vertex with repeated colour realises its own colour. Thus to make the colouring a b-chromatic one, we assign a proper colouring to $\mathrm{v}_{\mathrm{i}, \mathrm{j}}$ 's as follows. For $\mathrm{i}=1,2, \ldots, \mathrm{n}$ and $\mathrm{i} \equiv 2,3,4,(\bmod 5)$ assign the colour $c_{i-\left(\left[\frac{i}{5}\right]-1\right)}$ to the vertex $\mathrm{v}_{\mathrm{i}, \mathrm{i}+1}$ otherwise assign the colour $c$ to $\mathrm{v}_{\mathrm{i}, \mathrm{i}+1}$. Now the only vertex remaining to be coloured is $\mathrm{v}_{\mathrm{n}}$. Suppose we assign a new colour to the vertex $v_{n}$, the vertex does not realises the new colour, because $v_{n}$ is not adjacent with $v_{n-1}$ and $v_{i}$. Thus to realise the new colour we should colour the two neighbours of $v_{n}$ as $c_{n-1}$ and $c_{1}$, but by previous colouring, no vertex is left to be coloured. Thus introducing a new colour to the vertex $\mathrm{v}_{\mathrm{n}}$ is not possible. Note that any rearrangement of colours to the graph also fails to accomodate the new colour. Hence by colouring procedure this is a b-chromatic colouring and furthermore it is the maximum colouring possible.

\section{Example}
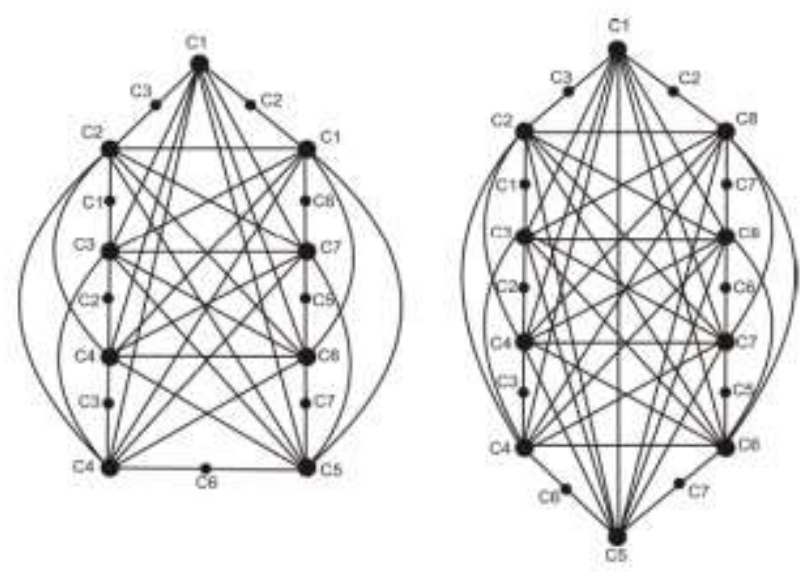

Figure 2: $\varphi\left[\mathbf{C}\left(\mathbf{C}_{9}\right)\right]=7$

$\varphi\left[C\left(C_{10}\right)\right]=8$

\section{THE b-COLOURING OF C $\left(P_{n}\right)$}

\subsection{Theorem}

For any path $\mathrm{P}_{\mathrm{n}}$ of length $\mathrm{n} \geq 5, \mathrm{n}=5 x+\mathrm{r}$

$$
\varphi\left[\begin{array}{ll}
C & P_{n}
\end{array}\right]= \begin{cases}n-x+1 & \text { where } \mathrm{r}=4 \\
\mathrm{n}-x & \text { otherwise }\end{cases}
$$

\section{Proof}

Let $P_{n}$ be any path of length $n-1$ with vertices $v_{1}, v_{2}, \ldots, v_{n}$. Let $\mathrm{V}_{\mathrm{ij}}$ represents the newly introduced vertex in the edge connecting $v_{i}$ and $v_{j}$. Now in $C\left(P_{n}\right)$ we can see that the vertex $v_{i}$ is adjacent with all the vertices except the vertices $v_{i+1}$ and $v_{i-1}$ for $i=2,3$, $\ldots ., \mathrm{n}-1 . \mathrm{v}_{\mathrm{n}}$ is adjacent with all the vertices except $\mathrm{v}_{\mathrm{n}-1}$ and $\mathrm{v}_{1}$ is adjacent with all the vertices except $v_{2}$. Now consider a blind colouring of $\mathrm{C}\left(\mathrm{P}_{\mathrm{n}}\right)$ as follows. Assign the colour $\mathrm{c}_{\mathrm{i}}$ to $\mathrm{v}_{\mathrm{i}}$ for $\mathrm{i}=1$, $2, \ldots ., \mathrm{n}$ due to the above mentioned non-adjacency of $\mathrm{v}_{\mathrm{i}}$ 's this colouring will not be a b-colouring. Thus to make it a bcolouring, we should assign a proper colouring to $\mathrm{v}_{\mathrm{ij}}$ 's. Consider an internal vertex $v_{i}$ of $P_{n}$, but $v_{i}$ is not adjacent with $v_{i+1}$ and $v_{i-1}$. Thus to realise the colour $c_{i}$ we should colour $v_{i, i+1}$ as $c_{i-1}$ and $v_{i, i-1}$ as $c_{i+1}$. Thus $v_{i}$ will realise the colour $c_{i}$. Now take the vertex $v_{i+1}$, which is coloured as $c_{i+1}$. In order to realise the colour $c_{i+1}$, we should colour the two neighbours of $v_{i+1}$ as $c_{i+2}$ and $c_{i}$, but by the previous colouring $v_{i}$ had left out only one vertex namely $v_{i+2, i+3}$ to be coloured. Thus realisation of $c_{i+1}$ is not possible. Similarly this will occur for $\mathrm{v}_{\mathrm{i}-1}$ too. This shows that assigning different colours to $v_{i}$ is not possible i.e. there should be repetation of 
colours. For $n \equiv 0,1,2,3(\bmod 5)$ there are $\left[\frac{n}{5}\right]+1$ repetitions otherwise $\left\lceil\frac{n}{5}\right\rceil+1$ repetitions.

\section{Case: 1}

When $\mathrm{r}=4, \mathrm{i}=1,2, \ldots ., \mathrm{n}$ and $\mathrm{i} \equiv 0,1,2(\bmod 5)$ assign the colour $c_{i-\left[\frac{i}{5}\right]}$ to the vertex $\mathrm{v}_{\mathrm{i}}$ otherwise assign the colour $c_{i}$

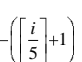

to the vertex $v_{i}$. Here also only the vertex with repeated colours realises its own colour. Thus to make the colouring a b-chromatic one, we assign a proper colouring to $\mathrm{v}_{\mathrm{ij}}$ 's as follows. For $\mathrm{i}=2,3$, $\ldots, \mathrm{n}-2$ and $\mathrm{i} \equiv 0,1,2,(\bmod 5)$ assign the colour $c_{i-\left(\left[\frac{i}{5}\right]+1\right]}$ to the vertex $\mathrm{v}_{\mathrm{ij}}$ otherwise assign the colour $c_{i+1-\left[\frac{i}{5}\right]}$ to the vertex $\mathrm{v}_{\mathrm{ij}}$. For remaining $\mathrm{v}_{\mathrm{ij}}$ 's we can assign any already assigned colours. Now all $v_{i}$ 's for $i=1,2, \ldots, n$ realises its own colour $c_{i}$. Hence by colouring procedure it is the maximum colouring.

\section{Case: 2}

When $\mathrm{r} \neq 4$, for $\mathrm{i}=1,2, \ldots, \mathrm{n}$ and $\mathrm{i} \equiv 0,1,2(\bmod 5)$ assign the colour $c_{i-\left[\frac{i}{5}\right]}$ to the vertex $\mathrm{v}_{\mathrm{i}}$ otherwise assign the colour $C_{i-}\left(\left[\frac{i}{5}\right]\right]_{+1}$

to the vertex $v_{i}$. Here also only the vertex with repeated colours realises its own colour. Thus to make the colouring a b-chromatic one we assign a proper colouring to $\mathrm{v}_{\mathrm{ij}}$ 's as follows. For $\mathrm{i}=2,3$, ..., $\mathrm{n}-3$ and $\mathrm{i} \equiv 0,1,2(\bmod 5)$ assign the colour $C_{i-\left(\left[\frac{i}{5}\right]\right]_{+1}}$ to the vertex $\mathrm{v}_{\mathrm{ij}}$ otherwise assign the colour $C_{i+1-}\left(\left[\frac{i}{5}\right]\right]$ to the vertex $\mathrm{v}_{\mathrm{ij}}$ and the remaining $\mathrm{v}_{\mathrm{ij}}$ otherwise assign the colour $c_{i+1-\left(\left[\frac{i}{5}\right]\right)}$ to the vertex $\mathrm{v}_{\mathrm{ij}}$ and the remaining $\mathrm{v}_{\mathrm{ij}}$ 's can be coloured with already used colours. Now the only vertex remaining is to colour $v_{n}$. Suppose we assign a new colour to the $v_{n}$, the vertex does not realises the new colour because $u_{n}$ is not adjacent with $v_{n-1}$. Thus to realise the new colour we should colour the neighbour of $v_{n}$ as $\mathrm{c}_{\mathrm{n}-1}$, which is not possible by colouring procedure. Thus introducing a new colour to the vertex $v_{n}$ is not possible. Note that any rearrangement of the colours to the graph also fails to accomodate the new colour. Hence by colouring procedure this is a b-chromatic colouring and furthermore it is the maximum colouring possible.

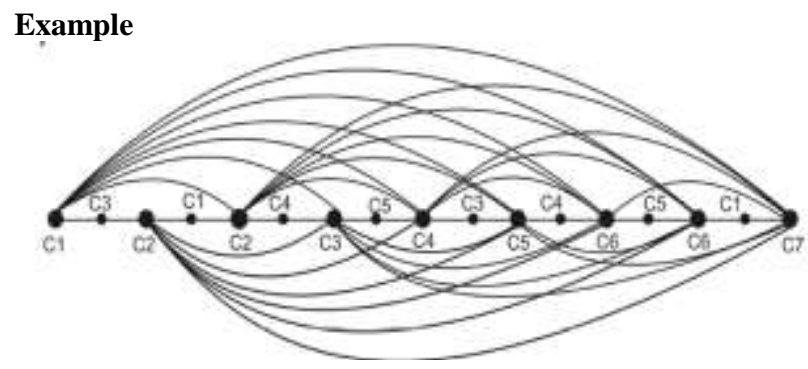

Figure 3 : $\varphi\left[\mathbf{C}\left(\mathbf{P}_{9}\right)\right]=7$

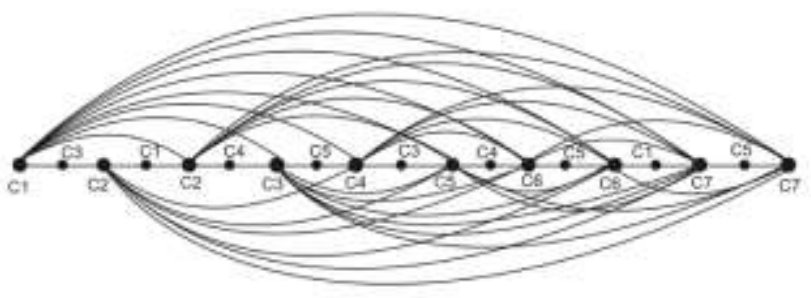

Figure $4: \varphi\left[\mathbf{C}\left(\mathbf{P}_{10}\right)\right]=7$

\section{REFERENCES}

(1) R. Balakrishnan, S. Francis Raj, Bounds for the b-chromatic number of the Mycielskian of some families of graphs, manuscript.

(2) D. Barth, J. Cohen, T. Faik, On the b-continuity property of graphs, Discrete Appl. Math. 155(2007)1761 - 1768.

(3) S. Corteel. M. Valencia-Pabon, J-C. Vera, On approximating the b-chromatic number. Discrete Appl. Math. 146(2005)106- 110 .

(4) B. Effantin, The b-chromatic number of-power graphs of complete caterpillars, J. Discrete Math. Sci. Cryptogr. $8(2005) 483-502$.

(5) C.T. Hoang, M. Kouider, On the b-dominating colouring of graphs, Discrete Appli. Math. 152(2005)176- 186.

(6) R.W. Irving, D.F. Manlove, The b-chromatic number of a graph, Discrete Appl. Math. 91(1999)127- 141.

(7) M. Kouider, A. El Sabili, About b-colouring of regular graphs, Rapport de Recherche No 1432, CNRS-Universite Paris Sud-LRI, 02/2006.

(8) K. Thilagavathi and N.Roopesh, "Generalization of Achromatic colouring of Central graphs", Electronic Notes in Discrete Mathematics 147-152, 33, 2009.

(9) K. Thilagavathi and N. Roopesh," Achromatic Colouring of Line graphs of Central graphs", Proceedings of the International Conference on Mathematics and Computer Science, 42-45, 2009.

(10) K. Thilagavathi and N. Roopesh, "Achromatic Colouring of $\mathrm{C}(\mathrm{Cn}), \mathrm{C}(\mathrm{Km}, \mathrm{n}), \mathrm{C}(\mathrm{Kn})$ and Spilt Graphs", Proceedings of the International Conference on Mathematical and Computer Science (ICMCS), 158-161, 2007. 